\title{
Clustering in complex networks. II. Percolation properties
}

\author{
M. Ángeles Serrano ${ }^{1}$ and Marián Boguñá ${ }^{2}$ \\ ${ }^{1}$ School of Informatics, Indiana University, Eigenmann Hall, 1900 East Tenth Street, Bloomington, Indiana 47406, USA \\ ${ }^{2}$ Departament de Física Fonamental, Universitat de Barcelona, Martí i Franquès 1, 08028 Barcelona, Spain
}

(Received 15 August 2006; published 28 November 2006)

\begin{abstract}
The percolation properties of clustered networks are analyzed in detail. In the case of weak clustering, we present an analytical approach that allows us to find the critical threshold and the size of the giant component. Numerical simulations confirm the accuracy of our results. In more general terms, we show that weak clustering hinders the onset of the giant component whereas strong clustering favors its appearance. This is a direct consequence of the differences in the $k$-core structure of the networks, which are found to be totally different depending on the level of clustering. An empirical analysis of a real social network confirms our predictions.
\end{abstract}

DOI: 10.1103/PhysRevE.74.056115

PACS number(s): 89.75.-k, 87.23.Ge, 05.70.Ln

\section{INTRODUCTION}

The general framework introduced in Paper 1 [1] to analyze clustering in complex networks provide us with the necessary tools to tackle the study of the percolation properties of clustered networks. The introduction of clustering in percolation analysis represents a theoretical challenge due to the fact that previous analytical approaches, applied to random two-point correlated directed and undirected networks [2-10], are based on the idea of branching process. One starts from a given vertex and follows all the edges attached to it. Then, the process is repeated again starting from the neighboring vertices avoiding the source one. All the vertices reachable from a given one belong to the same connected component and the network is said to be in the percolated phase when the largest component reaches proportions of the order of the network size, becoming the so-called giant connected component (GCC). This scheme works well when the network is locally treelike and thus the clustering coefficient is very small. Real networks, however, are shown to have a significant level of clustering-even for very large networks - that may change the percolation properties significantly.

Aside from the theoretical importance of percolation itself, there is a significant practical interest. Percolation is strongly related to epidemic processes. In fact, the simplest model for epidemic spreading, the susceptible-infectedremoved model $[11,12]$, can easily be mapped into a bond percolation problem [13-16]. In its simplest formulation, an infected individual becomes infected for a random time $t_{r}$ following a Poisson process, that is, $\psi_{r}\left(t_{r}\right)=\delta e^{-\delta t_{r}}$. On the other hand, when an infected individual is in contact with a susceptible one, it takes a random time $t_{i}$ to infect it, this process being a Poisson process as well, that is, $\phi_{i}\left(t_{i}\right)$ $=\lambda e^{-\lambda t_{i}}$. The probability that an infected individual infects a susceptible neighbor before it becomes removed is then

$$
p_{\text {inf }}=\int_{0}^{\infty} \phi_{i}(t) \Psi_{r}(t) d t=\frac{\lambda}{\lambda+\delta},
$$

where $\Psi_{r}(t)=\int_{t}^{\infty} \psi_{r}(\tau) d \tau$ is the probability that the vertex remains infected for a time larger than $t$. Since the infection uses the network as a template to spread, the infection process can be understood as a bond percolation problem over the original network when each edge is removed with probability $q_{\text {inf }}=1-p_{\text {inf }}$. The percolation threshold corresponds in this mapping to the onset of pandemic infections whereas the size of the giant connected cluster corresponds to the number of infected individuals.

In this second paper, we present analytical results for percolation in random networks with weak transitivity, that is, with degree-dependent clustering coefficient $\bar{c}(k)$ below $(k-1)^{-1}$, which extends and completes material previously published in [17]. In the case of percolation in the presence of strong transitivity-with $\bar{c}(k)>(k-1)^{-1}$ in a given domain-we present here interesting counterintuitive results which demonstrate that, in the percolation process, strong clustering favors the onset of the giant component whereas weak clustering hinders it. Furthermore, we show how these outcomes explain previous results by other authors $[18,19]$. We also discuss that these properties are intimately related to the structural organization of networks, in particular to their $k$-core decomposition [20,21]. We end this paper by taking a look at the pretty-good-privacy (PGP) web of trust [22], a large social network which turns out to be a nice example of a real system where our predictions apply.

\section{PERCOLATION IN WEAKLY CLUSTERED NETWORKS}

The standard percolation formalism based on branching processes overcounts the size of a node's second neighborhood when clustering is present. To correct for this effect, the usual procedure can be modified in the following way. One starts from a given vertex and follows all its edges. However, once placed in one of the neighbors, the next edges to follow are those not pointing to the neighborhood of the source vertex [23] (the edges pointing to the neighborhood of the source vertex are the ones responsible for clustering). It is worthwhile to notice that, even in this scheme, we are neglecting the fact that higher order loops may be present in the network. In particular, squares will connect vertices in the first neighborhood with a common vertex in the second neighborhood, overcounting it. Besides, when the multiplicity of the edges is large-by multiplicity $m$ we mean the number of triangles passing through an edge-squares induced by the merge of two triangles that share an edge appear. Therefore the implementation of the strategy out- 
lined above will become exact in the case of disjoint triangles but it will represent an upper bound to the real size of the giant component when triangles jam into edges. As explained in the preceding paper [1], weakly clustered networks are those which have a degree-dependent clustering coefficient $\bar{c}(k)<(k-1)^{-1}, \forall k$, implying that edge multiplicity is small and triangles disjoint. In contrast, strongly clustered networks have high edge multiplicity and thus triangles are forced to share edges.

With this in mind, let us start by defining the probability that a given vertex has $s$ reachable vertices, $G(s)$. For very heterogeneous networks this function is not very representative and we have to define the same probability but conditioned to the degree of the source vertex $G(s \mid k)$. These two functions are trivially related through

$$
G(s)=\sum_{k} P(k) G(s \mid k),
$$

where $P(k)$ is the degree distribution. Finally, we need to introduce an extra function, $g(s \mid k)$, which measures the probability that a vertex can reach $s$ other vertices given that it is connected to a vertex $v$ of degree $k$, and that it cannot visit either $v$ or its neighborhood. This last condition guarantees that we do not overcount contributions due to triangles. In this way, functions $G(s \mid k)$ and $g(s \mid k)$ are related through

$$
G(s \mid k)=\sum_{s_{1}, \cdots, s_{k}} g\left(s_{1} \mid k\right) \cdots g\left(s_{k} \mid k\right) \delta_{s, 1+s_{1}+\cdots s_{k}}
$$

We can find a recursion relation for $g(s \mid k)$ in the following way: we first compute the probability that the vertex under consideration, vertex $u$, has degree $k^{\prime}$ given that it was reached from a vertex of degree $k$, vertex $v$, using an edge of multiplicity $m$. In this way, the number of neighbors of $u$ that are not $v$ or neighbors of $v$ is $k^{\prime}-m-1$. Then, the number of reachable vertices from vertex $u$ is 1 (for itself) plus the number of vertices reachable from the $k^{\prime}-m-1$ available connections. This procedure can be repeated iteratively leading to a branching process with the constraint that at each generation point we can only use the free edges. Using this argument, function $g(s \mid k)$ can be written as

$$
\begin{aligned}
g(s \mid k)= & \sum_{k^{\prime}} \sum_{m} P\left(k^{\prime} \mid k\right) \phi\left(m \mid k k^{\prime}\right) \\
& \times \sum_{s_{1}, s_{2}, \cdots} g\left(s_{1} \mid k^{\prime}\right) g\left(s_{2} \mid k^{\prime}\right) \cdots g\left(s_{k^{\prime}-m-1} \mid k^{\prime}\right) \\
& \times \delta_{s, 1+s_{1}+s_{2}+\cdots s_{k^{\prime}-m-1}},
\end{aligned}
$$

where $P\left(k^{\prime} \mid k\right)$ is the probability that a vertex of degree $k$ is connected to a vertex of degree $k^{\prime}$ [29] and where we have defined $\phi\left(m \mid k k^{\prime}\right)$ as the probability that an edge connecting two vertices of degrees $k$ and $k^{\prime}$ has multiplicity $m$. The multiplicity matrix $m_{k k^{\prime}}$ can be computed as the first moment of $\phi\left(m \mid k k^{\prime}\right)$, i.e.,

$$
m_{k k^{\prime}}=\sum_{m=0}^{m_{k k^{\prime}}^{c}} m \phi\left(m \mid k k^{\prime}\right),
$$

where $m_{k k^{\prime}}^{c}=\min \left(k, k^{\prime}\right)-1$. For randomly assembled networks, we can make use of the probabilistic interpretation of the edge clustering coefficient $\bar{c}\left(k, k^{\prime}\right)=m_{k k^{\prime}} / m_{k k^{\prime}}^{c}[1,24]$ and write that

$$
\phi\left(m \mid k k^{\prime}\right)=\left(\begin{array}{c}
m_{k k^{\prime}}^{c} \\
m
\end{array}\right)\left[\bar{c}\left(k, k^{\prime}\right)\right]^{m}\left[1-\bar{c}\left(k, k^{\prime}\right)\right]^{m_{k k^{\prime}}^{c}} .
$$

In principle, this particular form of the distribution of $m$ is to be taken as an approximation. However, as we will see, in the case of randomly assembled networks it works extremely well.

We define the generating function of $g(s \mid k)$ as

$$
\hat{g}(z \mid k) \equiv \sum_{s} z^{s} g(s \mid k) .
$$

Using this transformation, Eq. (4) reads

$$
\hat{g}(z \mid k)=z \sum_{k^{\prime}} \sum_{m} P\left(k^{\prime} \mid k\right) \phi\left(m \mid k k^{\prime}\right)\left[\hat{g}\left(z \mid k^{\prime}\right)\right]^{k^{\prime}-m-1},
$$

which is a closed set of equations for the functions $g$ 's. Finally, $G(s \mid k)$ and $g(s \mid k)$ are related through

$$
\hat{G}(z \mid k)=z[\hat{g}(z \mid k)]^{k} .
$$

The percolation transition takes place when Eq. (8), evaluated at $z=1$, admits as a stable solution $\hat{g}(z=1 \mid k)=\xi(k) \leqslant 1$, that is, there is a finite probability $[1-\xi(k)]$ that the branching process extends up to infinity, meaning that a giant connected component has been formed. Since $\hat{g}(z=1 \mid k)=1$ is always a fixed point of Eq. (8), the onset of the giant component is the point at which this solution becomes unstable. To perform the stability analysis, we linearize Eq. (8) by plugging in a solution of the form $\hat{g}(z=1 \mid k) \approx 1+\chi(k) \epsilon$. In the limit $\epsilon \rightarrow 0$, this operation yields

$$
\chi(k)=\sum_{k^{\prime}}\left(k^{\prime}-1-m_{k k^{\prime}}\right) P\left(k^{\prime} \mid k\right) \chi\left(k^{\prime}\right) .
$$

The critical percolation point is ruled by the maximum eigenvalue $\Lambda_{m}$ of the matrix $\left(k^{\prime}-1-m_{k k^{\prime}}\right) P\left(k^{\prime} \mid k\right)$. When $\Lambda_{m}>1$, the solution $\hat{g}(z=1 \mid k)=1$ becomes unstable leading the network to the percolated phase, in which a macroscopic fraction of the system becomes globally connected. The relative size of the GCC can then be computed as

$$
\operatorname{gcc}=1-\left\langle[\xi(k)]^{k}\right\rangle .
$$

To be able to derive an analytic expression for the percolation condition, we have to assume some simplifications at this point. On one hand, we take $m_{k k^{\prime}}=m_{0}$, with $m_{0}$ a constant within the interval $[0,1]$, that is, we restrict to networks with weak transitivity. In this situation, making use of the closure condition derived in the preceding paper, 


$$
\sum_{k^{\prime}} m_{k k^{\prime}} P\left(k, k^{\prime}\right)=k(k-1) \bar{c}(k) \frac{P(k)}{\langle k\rangle},
$$

we can see that the degree-dependent clustering coefficient takes the form $\bar{c}(k)=c_{0}\left(m_{0}\right)(k-1)^{-1}$, where $c_{0}\left(m_{0}\right)$ is a certain function of $m_{0}$ to be determined. The second simplification assumes the absence of two-vertex correlations but, when clustering is present, uncorrelation can be attained for all degree classes except for the degree class $k=1$ (see discussion in Paper I [1]). The reason is that vertices of degree $k=1$ cannot participate in triangles and therefore connections involving these vertices are forced to follow a different pattern. Therefore we assume that $P\left(k, k^{\prime}\right) \propto k k^{\prime} P(k) P\left(k^{\prime}\right)$ only for $k, k^{\prime}>1$, whereas it takes a different form when $k=1$ or $k^{\prime}=1$. Using this factorization together with the normalization condition and $\Sigma_{k^{\prime}} P\left(k, k^{\prime}\right)=k P(k) /\langle k\rangle$, the transition probability $P\left(k^{\prime} \mid k\right)$ can be found to be

$$
P\left(k^{\prime} \mid k\right)= \begin{cases}\frac{1-2 \frac{P(1)}{\langle k\rangle}+P(1,1)}{\left(1-\frac{P(1)}{\langle k\rangle}\right)^{2}} \frac{k^{\prime} P\left(k^{\prime}\right)}{\langle k\rangle} & k \wedge k^{\prime}>1 \\ \frac{P(1,1)}{P(1)}\langle k\rangle & k=1 \wedge k^{\prime}>1 \\ \frac{k^{\prime} P\left(k^{\prime}\right)}{\langle k\rangle} & k>1 \wedge k^{\prime}=1 \\ \frac{P(1,1)}{P(1)}\langle k\rangle & k=k^{\prime}=1,\end{cases}
$$

where $P(1,1)$ is the probability that a randomly chosen edge connects two nodes of degree 1 , which remains as the only parameter to be determined.

If clustering is absent, then $m_{0}=0$ and $P(1,1)$ can factorize as $P(1,1)=P^{2}(1) /\langle k\rangle^{2}$. Otherwise, if clustering is finite, we can assume that all edges that are not involved into triangles are realized in the most random form. In a random uncorrelated networks, the probability that an edge is connecting two vertices of degrees $k$ and $k^{\prime}, P\left(k, k^{\prime}\right)$, factorizes as $P\left(k, k^{\prime}\right)=k k^{\prime} P(k) P\left(k^{\prime}\right) /\langle k\rangle^{2}$. On the other hand, this joint degree-degree distribution can be expressed in terms of the number of connections between different degree classes, $E_{k k^{\prime}}$, and the total number of edges $E$ as $P\left(k, k^{\prime}\right)=E_{k k^{\prime}} / 2 E$. Therefore if $E_{c}$ is the number of edges of our original network participating in triangles, the expected number of edges between vertices of degree 1 will read $E_{11}=N^{2} P^{2}(1) / 2(E$ $\left.-E_{c}\right)$. This leads to the following expression:

$$
P(1,1)=\frac{E_{11}}{2 E}=\frac{P^{2}(1)}{\langle k\rangle^{2}} \frac{1}{1-E_{c} / E} .
$$

$E_{c}$ can be computed as

$$
E_{c}=\frac{1}{2} \sum_{i, j} a_{i j} \Theta\left(m_{i j}-1\right),
$$

where $\Theta\left(m_{i j}-1\right)$ is the Heaviside step function. This equation can now be rewritten as

$$
E_{c}=E \sum_{k, k^{\prime}=2} P\left(k, k^{\prime}\right)\left\langle\Theta(m-1) \mid k, k^{\prime}\right\rangle,
$$

where the last average is taken over the set of edges connecting nodes of degrees $k$ and $k^{\prime}$, that is,

$$
\left\langle\Theta(m-1) \mid k, k^{\prime}\right\rangle=\sum_{m=1} \phi\left(m \mid k k^{\prime}\right)=1-\phi\left(0 \mid k k^{\prime}\right),
$$

which can be computed using Eq. (6). Using this expression, combined with the two-point correlation function, Eqs. (13) and (14), we can write the following equation for $P(1,1) \equiv x$ :

$$
x^{2}-\left(\frac{\langle\phi\rangle^{\prime}}{1-\langle\phi\rangle^{\prime}}+\frac{2 P(1)}{\langle k\rangle}\right) x+\frac{P^{2}(1)}{\langle k\rangle^{2}\left(1-\langle\phi\rangle^{\prime}\right)}=0,
$$

where $\langle\phi\rangle^{\prime}$ is the average of $\phi\left(0 \mid k k^{\prime}\right)$ over the set of vertices of degrees larger than 1. $P(1,1)$ corresponds to the smallest solution of this quadratic equation. Finally, using Eq. (12), the clustering factor $c_{0}\left(m_{0}\right)$ can be written as

$$
c_{0}\left(m_{0}\right)=m_{0} \frac{1-2[P(1) /\langle k\rangle]+P(1,1)}{(1-[P(1) /\langle k\rangle])} .
$$

Using the results above, the maximum eigenvalue of the matrix $\left(k^{\prime}-1-m_{k k^{\prime}}\right) P\left(k^{\prime} \mid k\right)$ can be analytically computed, yielding a percolation condition given by

$$
\frac{\langle k(k-1)\rangle}{\langle k\rangle}>\left[1+c_{0}\left(m_{0}\right)\right] \frac{m_{0}}{c_{0}\left(m_{0}\right)}\left(1-\frac{P(1)}{\langle k\rangle}\right) .
$$

It is easy to prove that the right hand side of this equation is always larger than or equal to 1 . This means that weakly clustered networks percolate at a higher density of connections as compared to the unclustered ones. For very low clustering, this term converges to 1 and so we recover the percolation threshold of random networks with a given degree distribution.

There is a particular case in which the symbiosis of a specific form of the degree distribution and a weak clustering in the frontier, $\bar{c}(k)=(k-1)^{-1}$, maintains the critical point unchanged with respect to the unclustered classical random graph. This model is studied in detail in Ref. [18] as a natural extension of the Erdös-Rényi random graph [26,27], where each possible triangle among a fixed number of vertices is realized with a given probability. In the thermodynamic limit, and for sparse networks, all edges in the ensemble have fixed multiplicity, $m=1$, and the generating function of the resulting degree distribution is $\hat{P}(z)=\exp \left[\langle k\rangle\left(z^{2}-1\right) / 2\right]$. Since odd degrees are not present, $P(1)=P(1,1)=0$ and the critical condition Eq. (20) becomes $\langle k(k-1)\rangle /\langle k\rangle>2$ which, for this particular degree distribution translates into $\langle k\rangle>1$, that is, the same percolation condition that applies for the classical random graph. 


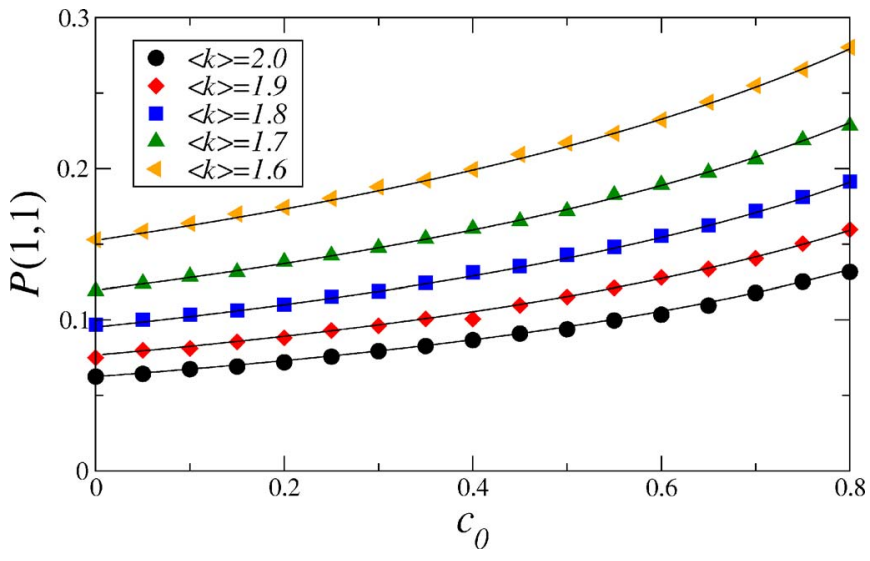

FIG. 1. (Color online) Values of the parameter $P(1,1)$ as a function of $c_{0}$ obtained from numerical simulations as compared to the analytical solution given by Eq. (18), for different values of the average degree and an exponential degree distribution. Symbols are numerical simulations using the algorithm of Ref. [25] and solid lines correspond to the analytical solution.

To check the accuracy of the formalism, we generate clustered random networks with the algorithm introduced in [25] with an exponential degree distribution and a degreedependent clustering coefficient of the form $\bar{c}(k)=c_{0}(k$ $-1)^{-1}[30]$. Notice that, when generating the networks, we fix the value of $c_{0}$ and not $m_{0}$, as it is done in the derivation above. However, we can make use of Eq. (19) to go from one parameter to the other. Figure 1 shows a perfect agreement between the empirical values for the parameter $P(1,1)$ as a function of $c_{0}$ for different values of the average degree and the analytical results derived from Eq. (18). In Fig. 2, we compare the relative size of the giant connected component as a function of $c_{0}$ with the numerical solution of Eqs. (8) combined with the transition probability given in Eq. (13). As it can be seen in the figure, the effect of clustering is to reduce the size of the giant connected component. The effect

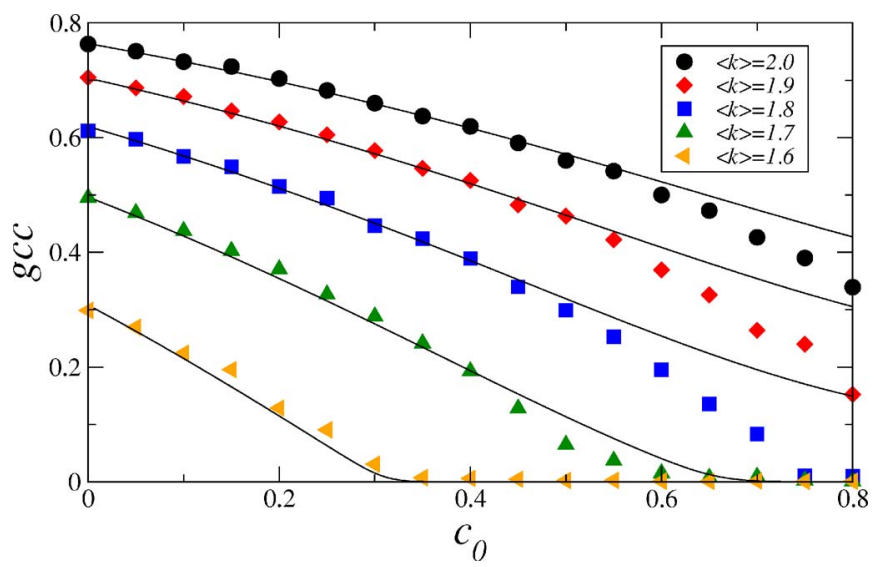

FIG. 2. (Color online) Relative size of the giant component in the case of $\bar{c}(k)=c_{0} /(k-1)$ as a function of $c_{0}$ for different values of the average degree. Symbols are numerical simulations using the algorithm of Ref. [25] for an exponential degree distribution. Solid lines correspond to the numerical solution of the set of equations (8). is so strong that, in networks with a moderate average degree, it completely fragments the network when $c_{0}$ exceeds a critical value. In other cases, the reduction of the size can be of more of the fifty percent. For values of $c_{0} \in[0,0.5]$, the agreement between our theory and numerical simulations is excellent. Beyond this point, our approximation slightly overestimates the GCC's size. This is mainly due to the fact that in this regime, links of multiplicity larger than 1 appear which, in turn, induces the presence of some loops of order 4.

\section{PERCOLATION IN STRONGLY CLUSTERED NETWORKS}

In the strong transitivity regime, the solutions obtained from our theory become upper bounds to the real size of the giant component due to the fact that then higher order loops appear. Unfortunately, it is not easy to extend the analytical calculations to this case but one can resort to numerical simulations. Fortunately, we can make use of the algorithm presented in Ref. [25], which allows to generate random networks with a given degree distribution, a fixed degreedependent clustering coefficient, and at the same time, to exert some control on the assortativity level of the network.

First, it is important to comment on the results by Newman [19], who solved exactly the bond percolation problem for the one-mode projection of random bipartite graphs. One of the main results in that study is that clustering, although makes the giant component smaller, favors its onset, which seems to be just the opposite result to the one that we obtained in the case of weak transitivity networks. To solve this puzzle, we first need to understand how one-mode projection networks are constructed. In a bipartite network, two types of vertices coexist, for instance scientists and scientific papers, with connections among them. The one-mode projection is then constructed by retaining just scientists and connecting them whenever they co-author the same paper. That means that all papers with more than two authors will give place to cliques of connected scientists. Therefore edges participating in triangles will have high multiplicity and the networks so generated will belong to the strong transitivity class. We shall see in the following that it is precisely the class the network belongs to-and not the scalar clustering coefficient - that determines whether clustering favors or not the onset of the giant component.

To check this issue, we have generated two networks with identical degree distribution (exponential with $\langle k\rangle=2$ ) and different forms for $\bar{c}(k)$, the first one with weak transitivity and the second one with strong transitivity. Then, we study their percolation properties by implementing a bond percolation experiment. We remove each edge with probability $q$ and measure the size of the giant component. Figure 3 shows the results of this program. As it is clearly seen, in both types of networks the size of the giant component is smaller than that of the unclustered network. However, the percolation threshold for the weak transitivity class is smaller than the unclustered value, whereas it is larger in the strong transitivity class, despite the fact that both networks have the same value for the scalar clustering coefficient $\bar{c}$. In Fig. 3, we also 

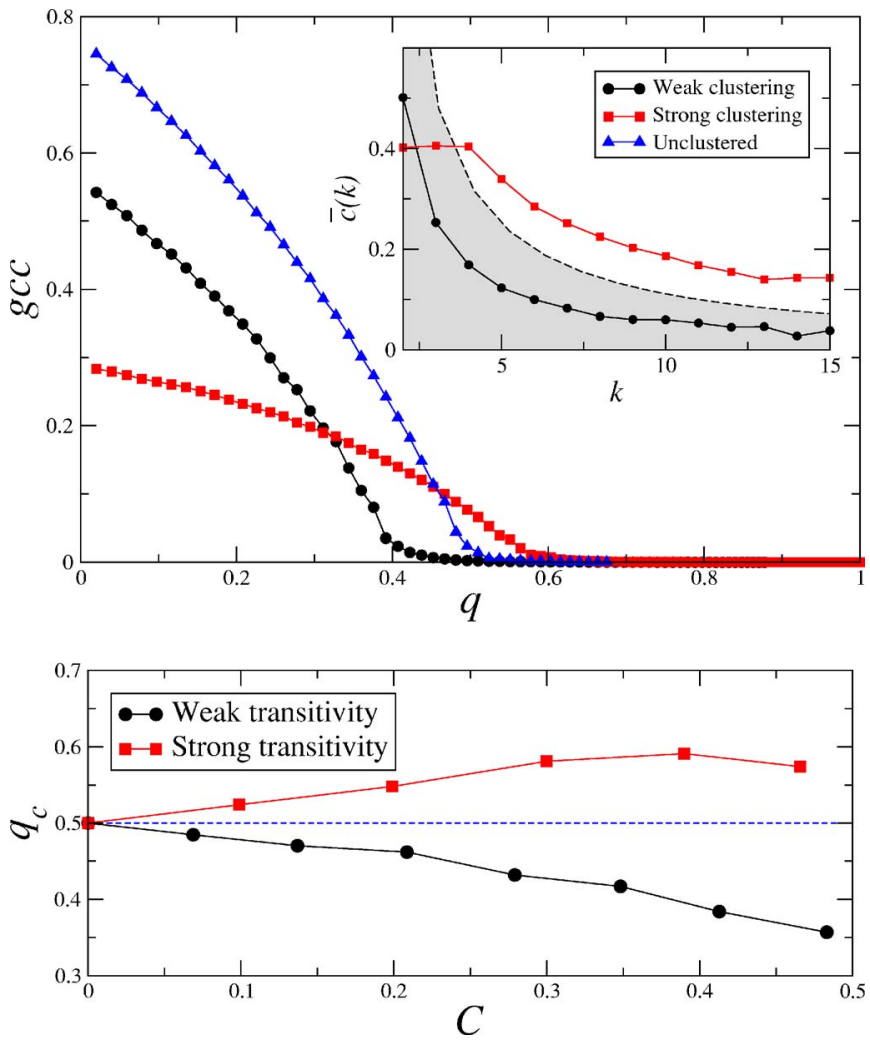

FIG. 3. (Color online) Top graph: Bond percolation on networks with exponential degree distribution $(\langle k\rangle=2)$ and weak clustering (black filled circles), strong clustering (red filled squares), and zero clustering (blue filled triangles). For both types of clustering the size of the giant component of the original networks is smaller than the unclustered one. However, the percolation threshold for the network with weak clustering is smaller than the unclustered net whereas it is larger for the network with strong clustering, despite the fact that both networks have the same value of $\bar{c}$. The inset shows the degree-dependent clustering coefficient for both networks. The area depicted in grey indicates the limits of weak clustering. Outside this region, the multiplicity of edges is necessarily larger than 1. Bond percolation simulations are performed over a single network of size $N=10^{5}$ and, then, averaged over 50 different realizations for each value of $q$. Bottom graph: Percolation threshold for strong and weak transitivity as a function of the global clustering $C=[1-P(1)]^{-1} \bar{c}$. The blue dashed line is the percolation threshold for the unclustered network $\left(q_{c}=1 / 2\right)$.

show the behavior of the percolation threshold $q_{c}$ for different values of the scalar clustering coefficient $C=\bar{c} /[1$ $-P(1)]$ (which is defined in the interval $[0,1]$ ) for the strong and weak transitivity cases, which confirms this trend.

Although illuminating, this analysis has the problem that we are comparing the resilience properties of networks with different giant components. Despite that the degree distribution is the same for all the networks, it could not be the case for their giant components, potentially changing then the location of the critical point. To overcome this problem, we generate a network with strong transitivity and extract its giant component. Then, using the degree distribution of this giant component we generate two more networks, one without clustering and the other one with weak

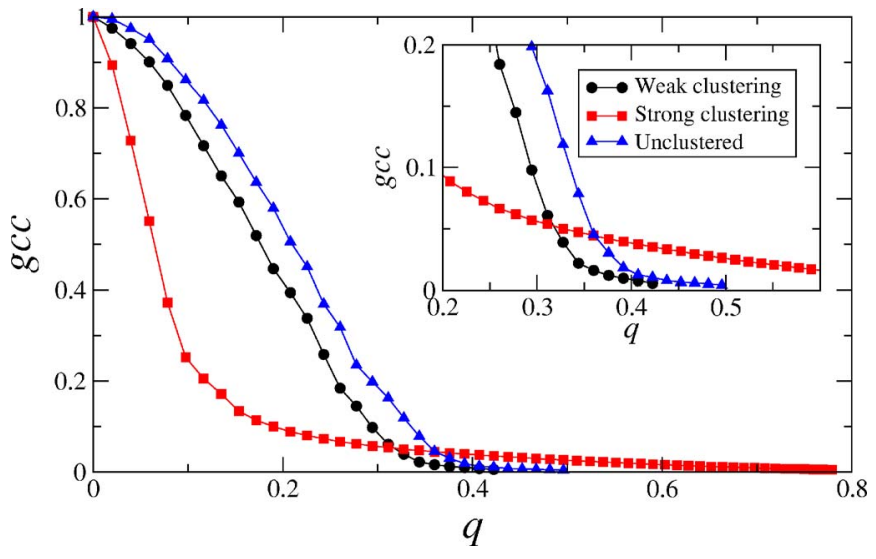

FIG. 4. (Color online) Results of the bond percolation process on networks with a degree distribution of the form $P(k) \sim k^{-\gamma}$, $\gamma=3.5$, and strong clustering (red filled squares), weak clustering (black filled circles), and unclustered (blue filled triangles). In all cases, the original networks are single connected components.

clustering. Finally, we check that these two networks are globally connected. We choose as the degree distribution to generate the strongly clustered network a function of the form $P(k) \sim k^{-\gamma}$, with $\gamma=3.5$. This particular form is convenient because using this distribution the range of degrees is relatively wide and, at the same time, the network still has a finite percolation threshold. We also restrict the degrees to values larger than two. Otherwise the unclustered network would not have a GCC. Figure 4 shows the results for the bond percolation process in these three networks. As it is clearly seen, the size of the GCC of the highly clustered network decreases very fast when a small fraction of edges is removed whereas the unclustered one is more resilient in this regime. However, as $q$ increases, the unclustered network undergoes the phase transition (at $q_{c}=0.4$ ) whereas the highly clustered net shows a surprising resilience, decaying very slowly as $q$ increases. On the other hand, the weakly clustered network is always less resilient than the unclustered one and undergoes the phase transition at a lower level of damage. These results confirm those shown in Fig. 3

To understand which is the origin of this behavior and the differences between weak and strong clustering, we need to understand the kind of structures that are formed depending on the clustering properties. The concept of $k$-core is particularly suitable for this purpose (see [21] for a very nice work on $k$-core percolation in random networks). The $k$-core of a network is the maximal subgraph such that all its vertices have $k$ or more connections within the subgraph. Therefore $k$-cores are subgraphs which are particularly resilient to the removal of edges if $k$ is large. In Fig. 5, we show the relative sizes of the giant $k$-cores of the networks of Fig. 4 as a function of $k$ compared with the cumulative degree distribution $P_{c}(k)=\sum_{k^{\prime} \geqslant k} P\left(k^{\prime}\right)$. This comparison is in order because $P_{c}(k)$ is the maximum possible value the giant $k$-core can attain. In the cases of weak clustering and unclustered network, the entire network forms a giant 2-core but the $k$-cores for $k>2$ do not exist. This result can be understood using results from [21] that state that, in random networks, the giant $k$-core undergoes a $k$-core percolation transition which 


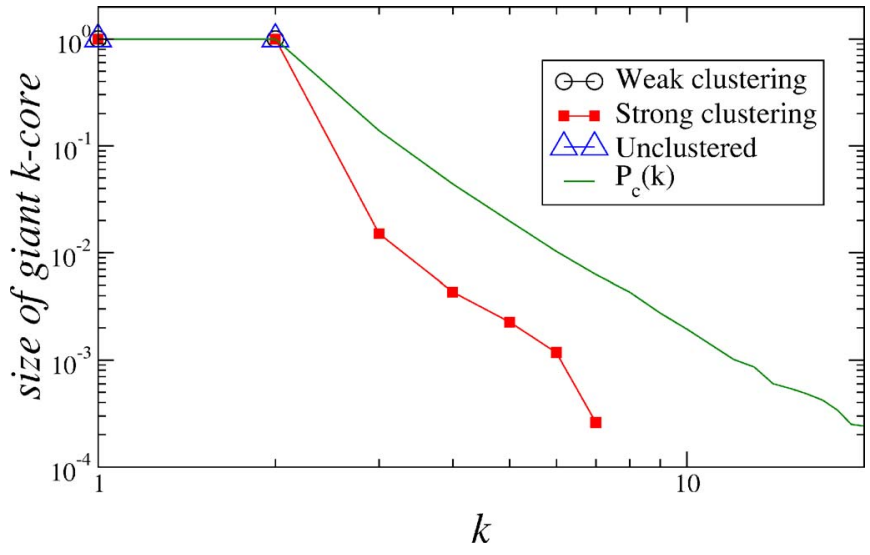

FIG. 5. (Color online) Relative sizes of the giant $k$-cores of networks with $P(k) \sim k^{-\gamma}$ and $\gamma=3.5$ for weak clustering, strong clustering, and unclustered, respectively.

is discontinuous, similar to what happens in first order phase transitions. In contrast, the strong clustered network has $k$-cores for $k>2$ which do not vanish, with a constant fraction of $P_{c}(k)$ belonging to them. This behavior extends up to degree $k=7$, and the $k$-core finally disappears at $k=8$. This result explains the strong resilience of the strongly clustered network since, although small, these $k$-cores with high $k$ are extremely difficult to break. This suggests that the properties of the critical percolation threshold in this class of networks should be tied to their $k$-core percolation properties. This is an issue that deserves further consideration and will be addressed in a future work.

\section{PERCOLATION IN SCALE-FREE CLUSTERED NETWORKS}

Random scale-free (SF) networks with $2<\gamma \leqslant 3$ have the peculiarity of not having a percolation threshold or, equivalently, $q_{c}=1$. This means that one has to remove the totality of the edges to break the network into disconnected components. In epidemiological language, it means that any disease, even a low infectious one, can propagate and infect macroscopic portions of the population. Given the important implications of this result, it is necessary to discern whether it can be applied to more general types of networks. For instance, it has been proved that two-vertices degree-degree correlations cannot restore a finite epidemic threshold $[9,28]$. However, this result cannot be applied to networks with high levels of clustering since the networks used in the demonstration had a vanishing clustering coefficient in the thermodynamic limit.

As we have already seen in previous sections, weak transitivity hinders the onset of the giant component, with a condition for its existence, in the uncorrelated case, given by Eq. (20) (that we repeat here for readability)

$$
\frac{\langle k(k-1)\rangle}{\langle k\rangle}>\left[1+c_{0}\left(m_{0}\right)\right] \frac{m_{0}}{c_{0}\left(m_{0}\right)}\left(1-\frac{P(1)}{\langle k\rangle}\right) .
$$

In the case of SF networks, the term on the left in this inequality diverges and therefore the condition is always ful-
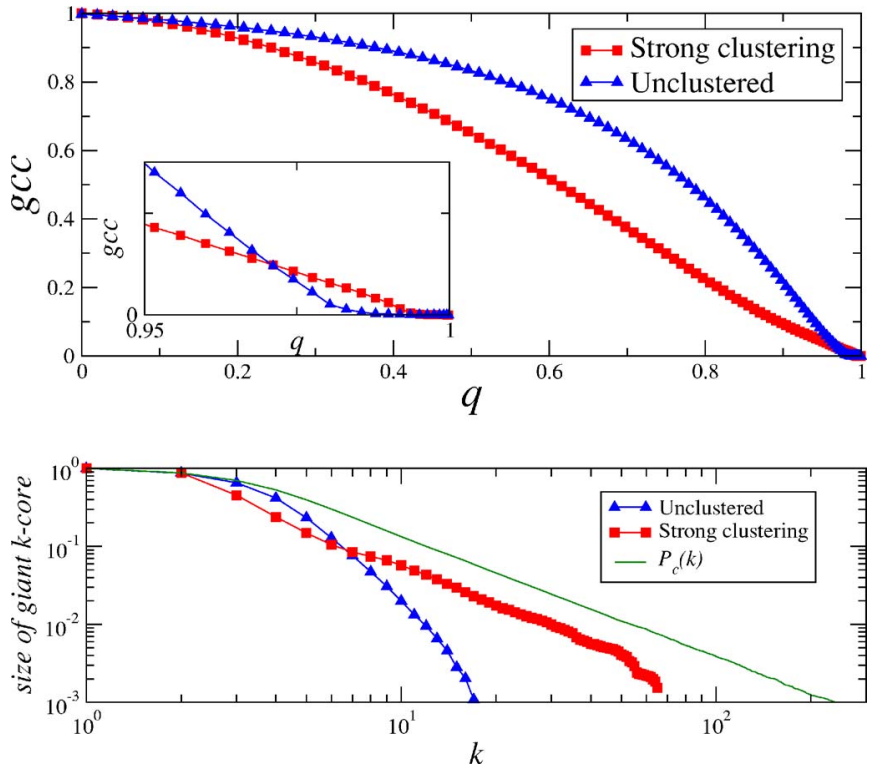

FIG. 6. (Color online) Top: results of the bond percolation process on a network with a degree distribution of the form $P(k)$ $\sim k^{-\gamma}$ and $\gamma=2.5$ and its randomized version. The inset shows a detail close to $q=1$. Bottom: relative sizes of the giant $k$-cores of these two networks. The solid line is the cumulative degree distribution $P_{c}(k)$.

filled. In short, this means that weak transitivity cannot restore a finite percolation threshold.

When the network belongs to the strong transitivity class, its percolation properties are not much different from networks with bounded fluctuations, except for the fact that the critical threshold is located at $q_{c}=1$. In Fig. 6, we repeat the same analysis performed in the previous section but now for a SF network. We first generate a highly clustered SF network with $\gamma=2.5$ and extract its giant component. Then, we randomize it to obtain an unclustered random network preserving the degree distribution. Finally, a bond percolation process is applied to both networks. As in the case of $\gamma=3.5$, the clustered network is less resilient than the unclustered one except for high levels of damage, where the clustered net is more resilient. In this case, the fact that we find a finite threshold is due to finite size effects. Again, the $k$-core analysis reveals a nested structure of $k$-cores up to $k=65$ following closely the shape of $P_{c}(k)$. In contrast, the $k$-cores for the unclustered network decays very fast as $k$ increases. These results imply strong transitivity cannot restore a finite percolation threshold either.

\section{PERCOLATION IN REAL NETWORKS: THE CASE OF THE PGP NETWORK}

We cannot end this paper without taking a look at the real world and checking if our results applies also there. To this end, we chose the pretty-good-privacy (PGP) web of trust analyzed in Ref. [22]. This is a nice example of a large social network based on trust. It arises as a consequence of the need for secure communications through the Internet. Without going into great detail, when a user A wants to send a message 

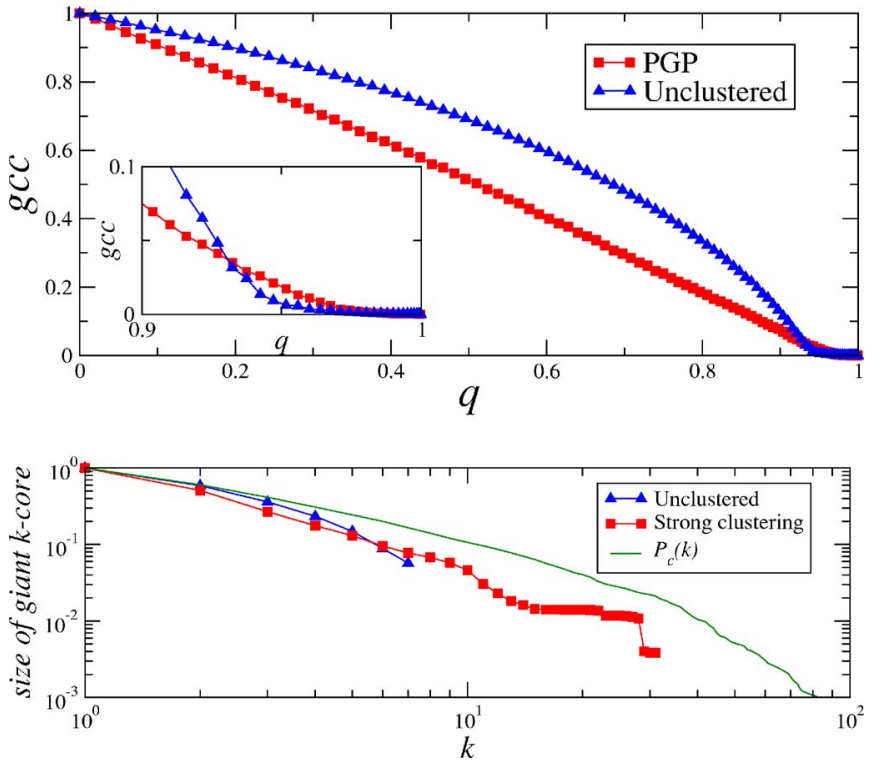

FIG. 7. (Color online) Top: bond percolation experiment performed on the giant component of the PGP network and on its randomized version. The inset shows a detail close to $q=1$. Bottom: relative sizes of the giant $k$-cores for the PGP network and its randomized version, respectively. The solid line is the cumulative degree distribution $P_{c}(k)$.

to another user B, she encrypts the message using the public key of user B who, afterwards uses her private key to decrypt it. In this way privacy is ensured. However, since everybody can generate his own pair of keys, it is not possible, in principle, to be sure that the person holding the key is who she claims to be. An imaginative solution to this problem is the web of trust. In this web, any user can sign the public key of another user, meaning that she trusts the other person is who she claims to be. This procedure generates a publicly available web of trust of users that have signed the public keys of other users. In principle, this web is directed. However, since we are interested in social ties, we filter out those connections that are not reciprocal. In this case, an edge among two persons is likely to represent a social relationship between them. After the filtering process, we obtain a network with $N=57243$ vertices and a giant connected component of $N_{g c c}=10680$ vertices. The network has a degree distribution with a heavy tail (although not a pure power law) extending up to $k \sim 200$ and a degree-dependent clustering coefficient which is constant up to degree $k=50$ followed by a sharp decay for $k>50$, with an overall value of 0.5 . This property sets the PGP network at the heart of the strong transitivity class.

The bond percolation experiment performed on the giant connected component of the PGP network reveals the same type of pattern that we have found before and is shown in Fig. 7. Again, the randomized version is more resilient except for very high values of $q$, where the PGP net is more resilient, with the critical point closer to 1 . The $k$-core decomposition also shows the same type of result. The PGP network has a nested $k$-core structure extending up to $k=31$, whereas the randomized network has only $k$-cores up to $k=7$.

We would like to stress that this behavior is by no means exclusive to the PGP network since many networks in the real world belong to the strong transitivity class. The results presented here are extremely relevant in the case of epidemic spreading. On the bad side, they suggest that real clustered networks are more prone to suffer epidemic outbreaks than unclustered networks. Yet, the relative size of the potentially infected population is smaller, which is indeed a positive result. We would also like to point out that the knowledge of the role that the giant $k$-cores have on the percolation properties could be used to design and plan more effective immunization strategies.

\section{CONCLUSIONS}

In this second paper, we have presented an analytical approximation to percolation in weakly clustered networks. Although this formalism is exact only in the limit of weak transitivity, it is an upper bound for the size of the GCC in all cases. Using this approach, we have seen that weakly clustered networks percolate at a higher density of connections as compared to the unclustered ones. By means of numerical simulations, we have also proved that the percolation threshold for networks in the weak transitivity class is smaller than the corresponding to an unclustered network with the same degree distribution. In contrast, this threshold is larger for nets in the strong transitivity class. This means that weak clustering hinders the appearance of the giant connected component whereas it is favored by strong clustering. To understand which is the origin of this behavior and the differences between weak and strong clustering, we have explored the structural organization of networks through their $k$-core decomposition, finding important differences among the two classes. In the case of scale-free networks, we have seen that neither weak nor strong transitivity can restore a finite percolation threshold. We have checked our results using a real social network, finding a very good agreement.

To summarize, in this paper and the preceding one we have developed a full theoretical approach to clustering in complex networks. We hope that these developments will improve the topological characterization of complex networks but also that they will help to produce a better understanding and a more realistic modeling of the dynamical processes that conform or use them.

\section{ACKNOWLEDGMENTS}

This work has been partially supported by DGES, Grant No. FIS2004-05923-CO2-02 and Generalitat de Catalunya Grant No. SGR00889. M.B. thanks the School of Informatics at Indiana University, where part of this work was developed. 
[1] M. A. Serrano and M. Boguñá, Phys. Rev. E 74, 056114 (2006).

[2] M. Molloy and B. Reed, Random Struct. Algorithms 6, 161 (1995).

[3] M. Molloy and B. Reed, Combinatorics, Probab. Comput. 7, 295 (1998).

[4] R. Cohen, K. Erez, D. ben-Avraham, and S. Havlin, Phys. Rev. Lett. 85, 4626 (2000).

[5] D. S. Callaway, M. E. J. Newman, S. H. Strogatz, and D. J. Watts, Phys. Rev. Lett. 85, 5468 (2000).

[6] M. E. J. Newman, S. H. Strogatz, and D. J. Watts, Phys. Rev. E 64, 026118 (2001).

[7] R. Cohen, D. ben-Avraham, and S. Havlin, Phys. Rev. E 66, 036113 (2002).

[8] M. E. J. Newman, Phys. Rev. Lett. 89, 208701 (2002).

[9] A. Vázquez and Y. Moreno, Phys. Rev. E 67, 015101(R) (2003).

[10] M. Boguñá and M. Á. Serrano, Phys. Rev. E 72, 016106 (2005).

[11] W. O. Kermack and A. G. McKendrick, Proc. R. Soc. London, Ser. A 115, 700 (1927).

[12] R. M. May and R. M. Anderson, Infectious Diseases of $\mathrm{Hu}$ mans: Dynamics and Control (Oxford University Press, Oxford, UK, 1991).

[13] P. Grassberger, Math. Biosci. 63, 157172 (1983).

[14] L. M. Sander, C. P. Warren, I. M. Sokolov, C. Simon, and J. Koopman, Math. Biosci. 180, 293 (2002).

[15] M. E. J. Newman, Phys. Rev. E 66, 016128 (2002).

[16] L. M. Sander, C. P. Warren, and I. M. Sokolov, Physica A 325, 1 (2003)
[17] M. A. Serrano and M. Boguñá, Phys. Rev. Lett. 97, 088701 (2006).

[18] X. Shi, L. A. Adamic, and M. J. Strauss, cond-mat/0605279 (unpublished).

[19] M. E. J. Newman, Phys. Rev. E 68, 026121 (2003).

[20] S. B. Seidman, Soc. Networks 5, 269 (1983).

[21] S. N. Dorogovtsev, A. V. Goltsev, and J. F. F. Mendes, Phys. Rev. Lett. 96, 040601 (2006).

[22] M. Boguñá, R. Pastor-Satorras, A. Díaz-Guilera, and A. Arenas, Phys. Rev. E 70, 056122 (2004).

[23] M. E. J. Newman, Soc. Networks 25, 83 (2003).

[24] F. Radicchi, C. Castellano, F. Cecconi, V. Loreto, and D. Parisi, Proc. Natl. Acad. Sci. U.S.A. 101, 2658 (2004).

[25] M. A. Serrano and M. Boguñá, Phys. Rev. E 72, 036133 (2005).

[26] P. Erdös and P. Rényi, Publ. Math. (Debrecen) 6, 290 (1959).

[27] P. Erdös and P. Rényi, Acta Math. Acad. Sci. Hung. 5, 17 (1960).

[28] M. Boguñá, R. Pastor-Satorras, and A. Vespignani, Phys. Rev. Lett. 90, 028701 (2003).

[29] The two-vertex probability distribution $P\left(k, k^{\prime}\right)$ is related to this transition probability by means of

$$
P\left(k, k^{\prime}\right)=\frac{k P(k)}{\langle k\rangle} P\left(k^{\prime} \mid k\right) .
$$

[30] To generate uncorrelated networks, we set $\beta=1$ in the algorithm of Ref. [25], which guarantees the factorization of $Q\left(k, k^{\prime}\right)[1]$ and hence the lack of degree-degree correlations other than the ones corresponding to degree 1 . 